\title{
Hole repair algorithm in hybrid sensor networks
}

\author{
Jian Liu ${ }^{1}$, Bo-Hao $\mathrm{Qu}^{1}$, Hong-Yan $\mathrm{Xu}^{1}$, Xiao-Wei Hu${ }^{1}$, Zhi-Qi Zhao ${ }^{2}$ and Yong Feng ${ }^{1}{ }^{1}$ * \\ ${ }^{I}$ School of Information, Liaoning University, Shenyang, China \\ ${ }^{2}$ Panjin High School, Panjin, China \\ E-mail: fengyong@lnu.edu.cn \\ *Corresponding author
}

\begin{abstract}
In Wireless sensor network, node error, energy depletion and other factors will lead to the appearance of hole which will cause network failure. In order to make the network more efficient, repair method based on the hybrid network model is proposed, namely activating a number of non-active nodes and calling mobile node to patching hole. This paper proposes two strategies: (1) Wake up the non-active nodes to reduce the hole area. It is proposed based on convex hull area reduction algorithm greedy algorithm for patching hole. (2) Call mobile node to fill hole gaps. Each mobile node covers more intersection arc of hole. The paper gives a Hole Repair Algorithm (HSNHRA, Hybrid Sensor Network Hole Repair Algorithm). Finally, the simulation results show the effectiveness of the proposed scheme, and the comparative analysis based on the experimental results shows the performance of the proposed scheme. It enables hole completely repaired, and the coverage and utilization of nodes have been improved.
\end{abstract}

Keywords: Hybrid Sensor Networks; Hole Repair Algorithm; Convex Hull; Mobile Node.

\section{Introduction}

Wireless sensor network is consists of a large number of sensor nodes which are composed as the self organization. It can be widely used in many fields [1], such as environmental monitoring, military, and anti-terrorism etc. Because the sensor network deploy randomly, network energy consume inequality, being attacked, fault and other reasons, which may cause some node death prematurely. Then hole can be formed in some part of network [2].

The research on hole patching in wireless sensor networks has already acquired some achievements. The representative are: Wang proposed the three distributed protocols of hole patching optimal target position by using Voronoi diagram to calculate the mobile node [3]. Yao proposed the HPA ( Hole Patching Algorithm ), the main idea of HPA is select a point along the perpendicular bisector between the boundary node of any two adjacent hole ,arrange a new node on the location to patch hole [4]. Wang and $\mathrm{Li}$ 
proposed strategy to using triangles based on mobile node one by one to repair hole [5]. Lam is proposed to realize the approximate uniform distribution of nodes in the sensor network by a large scale mobile node, which can eliminate the hole algorithm [6]. Sekhar proposed in the sensor network through the hole edge of the node to remove the hole of the algorithm [7]. Lu proposed Greedy Approximation Algorithm of Minimum Cover Set in Wireless Sensor Networks [8]. This paper consults his Greedy Algorithm to repair hole.

To realize complete hole patching, this paper propose a strategy for hole patching based on greedy algorithm (HSNHRA, Hybrid Sensor Network Hole Repair Algorithm).First of all according to the shape characteristic of hole chooses inactive nodes, active the node to patching hole. Then choose successor node according to the strategy, until the hole is completely patched. If not patched completely, calling the mobile node which can cover the most Arc intersection of hole to patching hole.

\section{Overview of Relevant Research}

\subsection{Hole in sensor network}

Assume that the adjacent nodes in sensor network can communicate each other, and the node itself has no GPS device. But one node can access the other's relative position information via the communication with adjacent node. a certain redundant non mobile node can be used to wake up to patching the hole, there is a certain mobile node can be used.

\subsection{Term}

Definition 1: Sensor network hole. In sensor network if exists a continuous area which is not be covered by any sensor node's monitoring area. This continuous region which can not be monitored is defined as sensor network hole.

Definition 2: Arc node of hole. In sensor network if a section of circular arc which is belong to the edge of a sensor node's perception range is not covered by any other sensor node's sensing area. The node is called arc node of hole. As shown in Figure 1 A, B, C, D, E, F, G, H are arc nodes of hole. They are adjacent each other and form a hole region.

Definition 3: Arc intersection of hole. In sensor network if there are two arc nodes of hole, they are sensing neighbor each other, and exists a intersection made by this two sensor nodes is not covered by other node's perception area. The intersection is called arc intersection of hole. As shown in Figure 1 the intersections of a, b, c, d, e, f, g, h are arc intersections of hole. 
Definition 4: Patching node. Use to patch the inactive node of hole. In Figure 1 node $\mathrm{I}$ is a patching node.

Definition 5: effective hole area: arc intersections of hole constitute the convex hull. The convex hull's area is the effective hole area, such as black solid lines in Figure 1, the convex polygon is hole convex hull area.

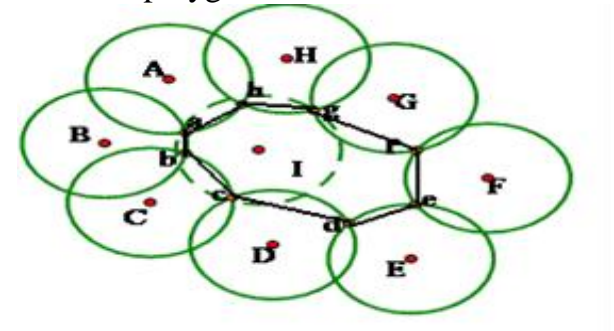

Fig. 1. Schematic diagram of hole and convex hull

\section{Hole Repair Algorithm in Hybrid Sensor Networks}

\subsection{Repair strategy based on Hybrid Sensor Networks}

Patching set can be obtained through the approach of set increment: add node to patching set continually, until the patching set cover the entire target area of hole. Suppose the target area of hole is $\mathrm{H}$, non-active nodes set is $\mathrm{S}$, mobile nodes set is $\mathrm{M}$, the strategy of constructing patching set $\mathrm{C}$ is as followed:

(1) Initial $\mathrm{C}=\varnothing$,

(2) Choose the suitable node s from $\mathrm{S}$, add it to $\mathrm{C}$, that is $\mathrm{C}=\mathrm{C} \cup\{\mathrm{s}\}$,

(3) If the area which is covered by $\mathrm{C}$ can patching $\mathrm{H}$, strategy stops and return $\mathrm{C}$, otherwise repeat step (2),

(4) if not patching the hole when the nodes in $\mathrm{S}$ is depleted, choose the suitable node in $M$, select the appropriate repair position, adding $C, C=C \cup\{m\}$,

(5) if the area covered by the $\mathrm{C}$ can be filled with $\mathrm{H}$ or $\mathrm{M}$ is empty, the policy ends to return to $C$; otherwise, repeat steps (4).

The key point in this strategy lies in step (2) and step (4): consider first step (2) HPA algorithm although always select the node with the maximum area covered, but filling efficiency is not high. Can be seen from Figure 2, the use of HPA algorithm to wake up nodes (dotted line), although the area to fill large, but the actual effect is not good because of a large number of gaps. To avoid the above problems, this paper in to fill a hole in the process, the introduction of greedy algorithm based on the consideration for hole convex hull and effective 
area decreased most, as shown in Figure 2, black solid line said nodes wake up, can make the convex hull area decreased most.

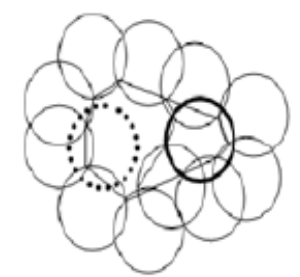

Fig. 2. Schematic diagram of patching lead to the convex hull area changes

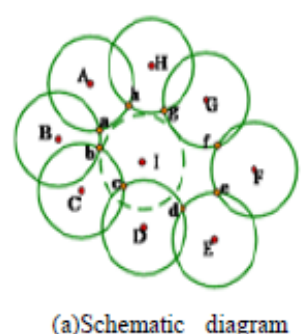

(a)Schematic diagram of (b)Schematic diagram of HPA to patch hole HSNHRA to patch hole

Fig. 3. Schematic diagram of HPA and HSNHRA o patch hole

Then we consider step (4): how a mobile node is added to fill the complement, from Figure 2, we can see, hole edge intersections must be concave points. These points using HPA algorithm it is difficult to cover. To avoid the above problems, this paper in to patching a hole in the process, the introduction of greedy algorithm based on account of preferential coverage arc intersection of hole, select the position where is covered most of arc intersection of hole, that can effectively prevent hole split, as shown in Fig. 3(a) shows that HPA algorithm will leave large amount split holes, and the use of this HSNHRA won't leave division hole, as shown in Fig. 3(b).

\subsection{Hybrid sensor network hole repair algorithm}

Input: non-active sensor node set $\mathrm{S}=\{\mathrm{s} 1, \mathrm{~s} 2, \ldots, \mathrm{sn}\}$, mobile node set $\mathrm{M}$ $=\{\mathrm{m} 1, \mathrm{~m} 2 \ldots \mathrm{mn}\}$ arc intersection set of hole: $\mathrm{A}$;

Output: hole patching set $\mathrm{C}$;

(1) $\mathrm{C}=\varnothing$; / / initialize

(2) While $(A \neq \varnothing \& \& S \neq \varnothing)$; / / if A is empty, hole has been patched

For each vi $\in \mathrm{S} / /$ vi is the node of $\mathrm{S}$

If (the number of arc intersections which are covered by vi $>0$ )

select vi which the effective area of the smallest hole

End for;

$\{$ Activate node vi; $\mathrm{C}=\mathrm{C} \cup\{$ vi $\} ;$ Update $\mathrm{A} ;\}$

End while;

If $(\mathrm{S}=\varnothing \| \mathrm{A} \neq \varnothing) / / \mathrm{S}$ set nodes are exhausted, holes not recovered 


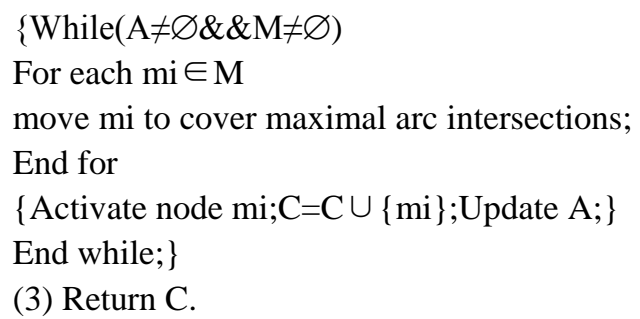

\section{Simulation Experiment}

Simulation experiment is carried out in a rectangular region of a $60 \mathrm{~m} \times 30 \mathrm{~m}$, randomly in the region deployed 20 sensors, the sensing radius of each sensor node is $8 \mathrm{~m}$, in the area of randomly generated a closed hole, the node distribution and the void hole as shown in Figure 4. Also shows according to HSNHRA deployment node coverage hole in which the dark area as the sensing range of the nodes.

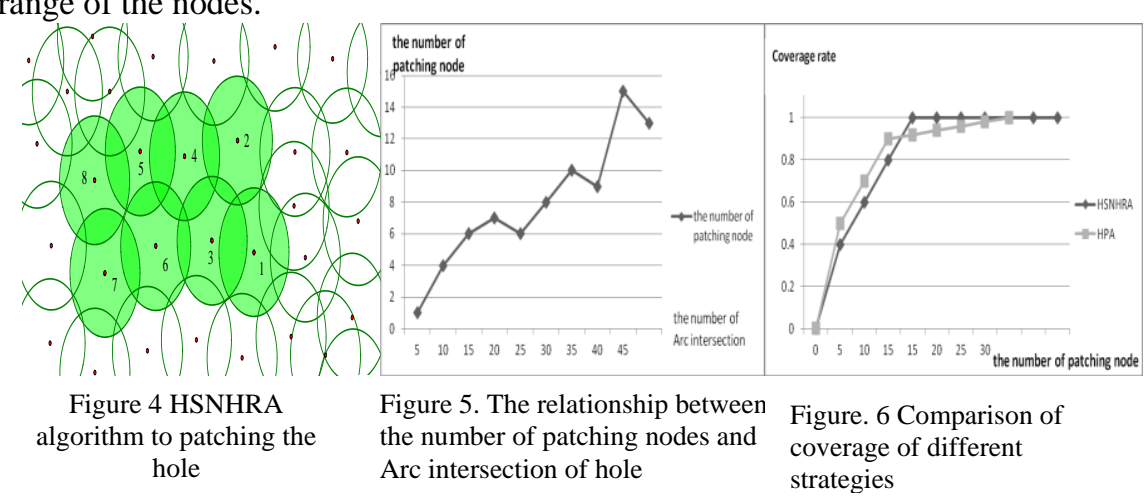

To better illustrate the number of nodes and arc intersections of hole, according to the HSNHRA strategy on randomly generated, area of different hole to fill, and statistics of the node number, corresponding points between the number of nodes and arc intersections of hole relationship as shown in Figure 5. As can be seen from Figure 5, the number of patching nodes not only with arc intersection of related, also with an hole geometry related. When the hole irregular shape, need more nodes to fill the holes, which further indicates that the importance of the geometric features of empty hole filling. Overall, they are positively correlated. 
In order to explain the advantages of this strategy, the paper compares the coverage of the HPA strategy with the literature [4]. As can be seen from Figure 6, HPA can achieve $80 \%$ of the node coverage faster than HSNHRA, but after $90 \%$, its node coverage increased slowly, and HSNHRA can reach $100 \%$ of the node coverage quickly. This is because in the HSNHRA each node coverage hole size is very close, and HPA strategy due to a large empty area covered, but will produce the large number of hole split, so HPA strategy after achieving a higher degree of coverage, $100 \%$ of the node coverage was very difficult.

\section{The Ending Word}

In this paper, we give a new method to repair the holes in the wireless sensor networks. According to the strategy deployment of nodes to patching the hole, first according to the geometric characteristics activate hole non active nodes in the best position to reduce the effective hole area, and mobile nodes deployment to coverage the most arc intersection of hole, so as to achieve the purpose of the least nodes deployment, and can effectively avoid hole split. At last, the performance of the strategy is analyzed by simulation experiment. The experimental results show that the strategy can improve the coverage and utilization of hole patching nodes.

\section{Acknowledgments}

Work partially supported by grant 12YJCZH048 of the Ministry of Education Research of Social Sciences Youth Fund Project and grant 2013020031 of the Natural Science Foundation of Liaoning Province of China.

\section{References}

1. I. F. Akyildiz, W. Su, Y. Sankarasubramaniam. Wireless sensor networks: A survey[J]. Computer Networks, 2002, 38(4): 393-422.

2. Wu Xiao-Bing Chen Gui-Hai The Energy Hole Problem of Nonuniform Node Distribution in Wireless Sensor Networks[J]. Chinese journal of computers, 2008, 31(2): 253-261.(In Chinese).

3. G. Wang, G. Cao. Movement-assisted sensor deployment[J]. IEEE Transactions on Mobile Computing, 2006, 5(6): 640-652.

4. J. X. Yao, G. Y. Zhang. Decentralized Detection and Patching of Coverage Holes in Wireless Sensor Networks[C]. Proc. of International Conference on Intelligent Sensing, Situation Management, Impact Assessment, and Cyber-Sensing, Orlando, Florida: SPIE, 7352: 73520V, 2009. 
5. L. M. Wang, F. Li, Y. Qin. Resilient method for recovering coverage holes of wireless sensor networks by using mobile nodes[J]. Journal on Communications, 2011, 32(4): 1-8.

6. M. L. Lam, Y. H. Liu. Two distributed algorithms for heterogeneous sensor network deployment towards maximum coverage[C]. Proceedings of the IEEE International Conference on Robotics and Automation, Pasadena, California, USA, 2008: 3296-3301.

7. A. Sekhar, B. S. Manoj. Dynamic coverage maintenance algorithms for sensor networks with limited mobility[C]. Proceedings of the 3rd IEEE International Conference on Pervasive Computing and Communications, Hawaii, 2005: 51-60.

8. K. Z. Lu, H. Y. Sun. Greedy Approximation Algorithm of Minimum Cover Set in Wireless Sensor Networks[J]. Journal of Software, 2010, 21(10): 2656-2665. 